\title{
Value Engineering Method for Selection of Gas Pipeline Materials
}

\author{
Jia-You LIU \\ Department of Resources and Civil Engineering, Shandong University of Science and Technology, \\ Tai'an271019, China \\ *liu_jiayou@163.com
}

Keywords: Value Engineering (VE), Gas Pipeline Materials, Function Analysis, Cost Analysis, Value Index.

\begin{abstract}
Selecting reasonable gas pipeline materials is of great significance to improve the safety and reliability of gas transmission and distribution system and reduce the project cost in gas engineering. A method used for selection of gas pipeline materials is presented used VE theory. In this method, a mathematical model to select the optimal gas pipeline material is founded. The program of the model includes four parts: research object determination, function analysis of gas pipeline materials, cost analysis and value analysis, and the function analysis is the most important part. The gas pipeline materials function indexes system is constructed to analyze the function of pipeline materials. The value index is treated as measurement parameter to evaluate the technical-economic characteristic of the pipeline materials. By studying an application case of gas engineering, the selected pipeline material is compliance with engineering practice, the method is proved to be feasible for gas pipeline materials selection in gas engineering.
\end{abstract}

\section{Introduction}

Gas pipeline is an important part of gas transmission and distribution system, and its basic function is to supply gas safely and reliably for different users. In gas engineering, the cost of pipeline is about $60 \% \sim 70 \%$ of the total project cost [1]. So selecting reasonable gas pipeline materials is of great significance to improve the safety and reliability of gas transmission and distribution system and reduce the project cost. At present, steel pipe (SP), ductile iron pipe (DIP), polyethylene pipe (PEP) and steel reinforced plastics pipe are commonly used in gas engineering, and rubber pipe, thin-walled stainless steel pipe, steel thread pipe and copper pipe, etc. are also used in indoor gas engineering. In general, gas pipeline material is selected through qualitative analysis and cost comparison [2]. Yang [3] suggested select gas pipe materials using fuzzy comprehensive evaluation method. The paper intends to establish a model to select the optimal gas pipeline material based on VE theory.

As a management theory, VE was developed by L. D. Miles who is an American electric engineer [4]. The theory has been widely used in lots of fields and achieved better profit [5]. And as a very effective theory of technical-economic analysis, the optimal value of the research object can be got by functions-cost analysis and ongoing innovation [6].

\section{Model of Pipeline Material Selection in Gas Engineering Used VE Method}

\section{VE Theory}

In VE theory, function analysis is the most important, and the aim of value analysis is to gain the optimal function with the least life cycle cost (LCC). The theory of VE can be expressed by Eq.(1).

$$
V=F / C
$$

Where, $V$ represents the research object index of value or value; $F$ represents the coefficient of function or function; $C$ represents the coefficient of cost or LCC. 
$V$ shows the interrelation between the function and the cost. The target of VE is to get the value as larger as possible with a reasonable lower LCC. To gain a higher $V$, it's necessary to reduce LCC and improve function within a reasonable range.

In general, the program of value analysis includes four parts: research object determination, function analysis, cost analysis and value analysis [5], [7].

\section{A Model for Pipeline Materials Selection in Gas Engineering Based on VE}

\section{Research Object Determination.}

Using VE theory to select gas pipeline material, the pipe materials should be considered as the research objects, and the pipe materials should be able to be applied in gas engineering.

\section{Function Analysis of Pipeline Materials.}

Function Index Determination.To ensure supplying gas reliably and safely for different users, gas pipeline materials must meet the requirements of pipe material characteristic, construction performance and service performance, etc. According to experts' suggestion and engineering practice, a structure diagram of pipeline materials' function indexes in gas supply engineering is constructed and shown in Fig.1.

Function Weight Determination.To gain the coefficient of functions of the pipeline materials, the function indexes' weights in Fig.1 are needed. According to structure diagram of pipeline materials, Analytic hierarchy process (AHP) method is adopted to gain the the indexes' weights [8], [9]. Firstly, based on the indexes' relative importance, a judgment matrix A expressed by Eq.(2) is constructed by using 9-level degree method (Table 1).

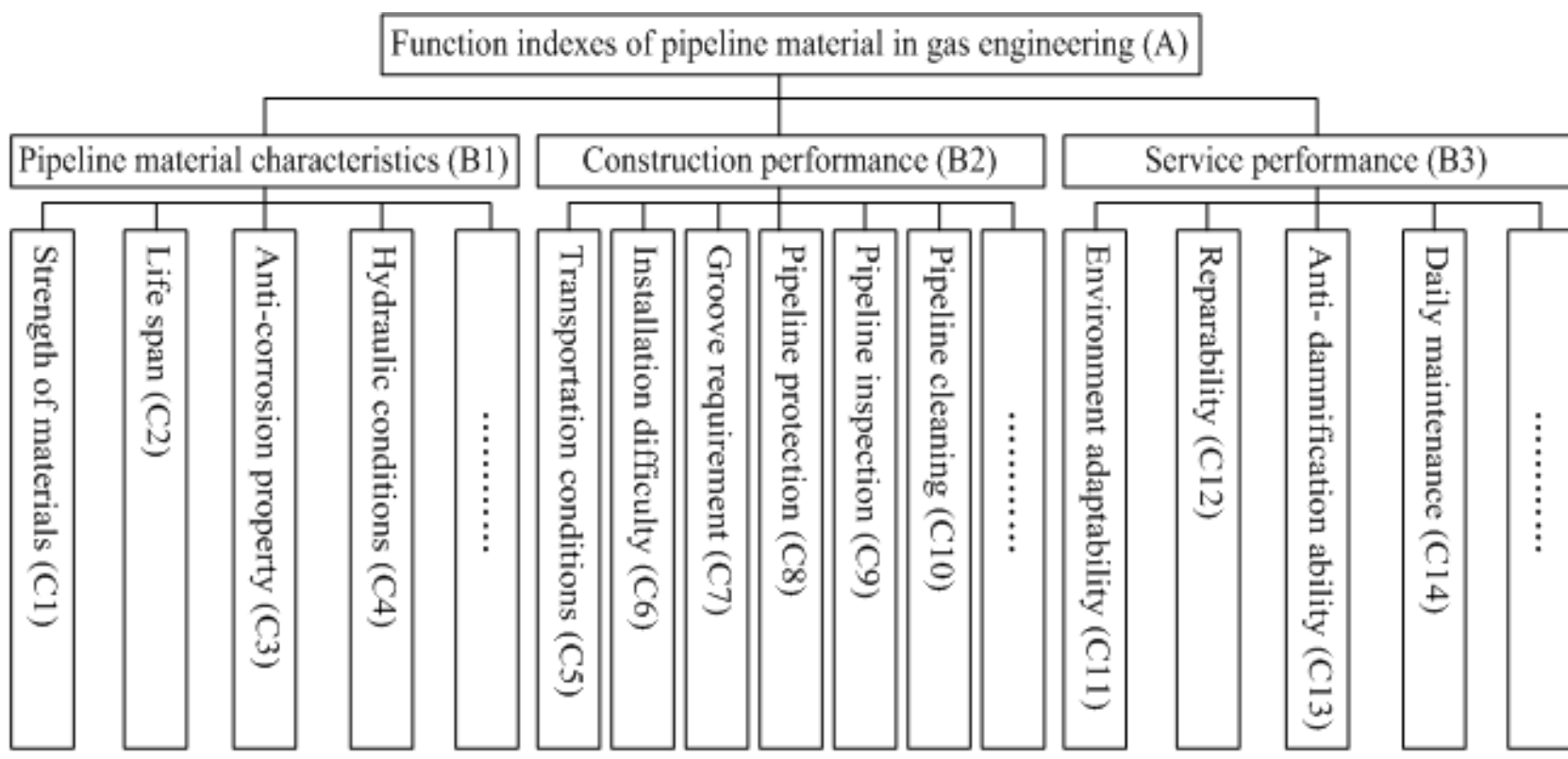

Fig.1 Gas Pipeline Materials Function Indexes System

$$
\begin{gathered}
A=(A)_{n \times n}=\left[\begin{array}{cccc}
a_{11} & a_{12} & \cdots & a_{1 n} \\
a_{21} & a_{22} & \cdots & a_{2 n} \\
\vdots & \vdots & \vdots & \vdots \\
a_{n 1} & a_{n 2} & \cdots & a_{n n}
\end{array}\right] \\
a_{i j}>0, a_{i i}=1, a_{i j}=1 / a_{j i},(i, j=1,2, \ldots, n)
\end{gathered}
$$

Where, $n$ represents the matrix order. 
Then, the matrix eigenvector $\left(W_{1}, W_{2}, \cdots, W \mathrm{n}\right)^{\mathrm{T}}$ and the maximal matrix eigenvalue $\lambda_{\max }$ can be calculated using Eqs.(3) (5).

Table 1. Principle of 9-level Degree Method

\begin{tabular}{|c|c|}
\hline Degree $a_{i j}$ & Meaning \\
\hline 1 & $a_{i}$ and $a_{j}$ are equally significant \\
\hline 3 & $a_{i}$ is a little significant than $a_{j}$ \\
\hline 5 & $a_{i}$ is distinctly significant han $a_{j}$ \\
\hline 7 & $a_{i}$ is greatly significant than $a_{j}$ \\
\hline 9 & $a_{i}$ is extremely significant than $a_{j}$ \\
\hline $2,4,6,8$ & The middle value of the upper degrees \\
\hline $\bar{W}_{i}=\sqrt[n]{\prod^{n} a_{i j}}$ & \\
\hline$W_{i}=\bar{W}_{i} / \sum^{n} \bar{W}_{i}$ & \\
\hline$\lambda_{\max }=\frac{1}{n} \sum_{i=1}^{n} \frac{(A W)_{i}}{W_{i}}$ & \\
\hline
\end{tabular}

Finally, beacause of the research object's complexity and people's knowledge's limitation, the judgment matrix consistency is requireded to test, and the consistency can be measured using consistency index and calculated by Eq.(6).

$$
C . I .=\frac{\lambda_{\max }-n}{n-1}
$$

Where, C.I. represents the matrix consistency index.

The consistency rate (C.R.) is gained by Eq.(7) using the average random consistency index (R.I.) (Table 2).

$$
C . R .=\frac{C . I .}{R \cdot I .}
$$

Table 2. Average Random Consistency Index (R.I.)

\begin{tabular}{ccccccccccc}
\hline$n$ & 1 & 2 & 3 & 4 & 5 & 6 & 7 & 8 & 9 & 10 \\
\hline$R . I$. & 0 & 0 & 0.58 & 0.90 & 1.12 & 1.24 & 1.32 & 1.41 & 1.46 & 1.49 \\
\hline
\end{tabular}

In general, if the matrix has the better consistency, C.R. $<0.1$, or it is required to modify the matrix.

Coefficient of Function Determinaion.According to the pipeline materials' function indexes shown in Fig.1, 10-points system is used to grade indexes based on the function level of satisfaction. The total grade is gained using the function indexes' weights and grades by Eq.(8), and the function coefficients are gained by Eq.(9), assuming there are $q$ function indexes and $p$ pipeline materials.

$$
G_{i}=\sum_{j=1}^{q} W_{j} G_{i j},(i=1,2, \ldots, p)
$$




$$
F_{i}=\frac{G_{i}}{\sum_{i=1}^{p} G_{i}},(i=1,2, \ldots, p)
$$

Where, $G_{\mathrm{i}}$ reprsents the $i$ pipeline material's total grade; $W_{\mathrm{j}}$ reprsents the the $j$ function index weight; $G_{\mathrm{ij}}$ reprsents the $j$ function index grade of the $i$ pipeline material; $F_{\mathrm{i}}$ reprsents the the $i$ pipeline material function coefficient.

Cost Analysis.

The LCC of pipeline material in gas engineering includes pipeline investment cost and operating cost. The annual cost of pipeline materials is calculated by annual cost method and the expression is Eq.(10).

$$
A C=P \cdot \frac{r \cdot(1+r)^{t}}{(1+r)^{t}-1}+O C
$$

Where, $A C$ reprsents annual cost; $P$ reprsents the pipeline material investment cost; $O C$ reprsents the pipeline material annual operating cost, $O C=d \cdot p ; d$ reprsents the proportion of depreciation and maintenance costs to pipeline investment cost; $r$ reprsents annual interest rate, taking $5 \%$; $t$ reprsents calculating period.

The pipeline material coefficient of cost can be gained by Eq.(11).

$$
C_{i}=\frac{A C_{i}}{\sum_{i=1}^{p} A C_{i}},(i=1,2, \ldots, p)
$$

Where, $C_{\mathrm{i}}$ reprsents the $i$ pipeline material cost coefficient; $A C_{\mathrm{i}}$ reprsents the $i$ pipeline material annual cost.

\section{Value Analysis.}

The value indexes of the pipeline materials can be gained utlizing the calculated coefficients of function and coefficients of cost by Eq.(1). According to the VE theory, the pipeline material with the largest value index has the necessary function with the least LLC, and it is the best pipeline material for the project.

\section{Application Case}

A mid-pressure gas pipeline network planning of some town is taken as an example. The gas supply quantity of the town is $10400 \mathrm{~m}^{3} / \mathrm{h}$. The gas source is dry natural gas and the designed gas supply pressure is $0.35 \mathrm{MPa}$. According to engineering practice and local conditions, SP, DIP and PEP can be used as the pipeline material of the mid-pressure pipeline. The structure of the pipeline network is identified. After the hydraulic calculation for the gas pipeline network [10], the required nominal diameters and lengths of each pipeline material are shown inTable 3.

Table 3. The Diameters and Lengths of Pipeline Materials

\begin{tabular}{cccc}
\hline Pipeline material & SP & DIP & PEP \\
\hline & $100 \times 4.06$ & $150 \times 4.06$ & $110 \times 4.06$ \\
Nominal diameter[mm] $\times$ length[km] & $150 \times 2.27$ & $200 \times 2.27$ & $160 \times 2.27$ \\
& $200 \times 1.01$ & $300 \times 1.01$ & $200 \times 1.01$ \\
& $250 \times 2.33$ & $400 \times 2.33$ & $250 \times 2.33$ \\
\hline
\end{tabular}


With the experts' suggestion, by comparing the function indexes of the pipeline materials each other, the indexes' weights in Fig. 1 are gained adopting AHP method and are shown in Table 4 and Table 5.

Table 4. Function Indexes Weights in B Level

\begin{tabular}{cccc}
\hline Index & B1 & B2 & B3 \\
\hline Weight & 0.6301 & 0.1515 & 0.2184 \\
\hline
\end{tabular}

Table 5. Function Indexes Weights in C Level

\begin{tabular}{cccccccc}
\hline Index & C1 & C2 & C3 & C4 & C5 & C6 & C7 \\
\hline Weight & 0.5018 & 0.2201 & 0.2201 & 0.0579 & 0.0610 & 0.4740 & 0.1805 \\
\hline Index & C8 & C9 & C10 & C11 & C12 & C13 & C14 \\
\hline Weight & 0.0976 & 0.1095 & 0.0775 & 0.1051 & 0.4457 & 0.2848 & 0.1644 \\
\hline
\end{tabular}

Based on the experts suggestion and engineering practice, the function indexes of the pipeline materials are graded, and the function grade and function coefficient can be gained by Eq.(8) and Eq.(9) and are shown in Table 6.

In gas engineering, pipeline investment cost includes pipe cost and construction cost, and operating cost which includes depreciation and maintenance costs of pipelins, etc. The annual cost and cost coefficient can be gained using Eq.(10) and Eq.(11) respectively, the value of $d$ is 3.5\% for SP and 2\% for DIP and PEP [11]. The costs are shown in Table 7.

The value indexes can be gained using the function coefficients in Table 6 and cost coefficients in Table 7 by Eq.(1), and the indexes are shown in Table 8.

As shown in Table 8, the PEP index of value is the largest, so PEP is the best pipeline material for the gas supply project. The function coefficient of SP is lower, but its cost is higher, so its value index is lower; the function coefficients of DIP and PEP are higher, but the cost of PEP is lower, so the value index of PEP is higher than that of DIP.

In practical engineering, especially in urban gas pipeline network planning, SP is widely used because of its higher strength, but its anti-corrosion property is poor, its life span is short, and its maintenance cost is higher, so when gas supply pressure is higher, SP is recommended to be used; DIP has the advantage of higher strength, good anti-corrosion property, but its cost is higher and gas is easier to leak when using DIP to supply dry gas. PEP is recommended to be used as gas pipeline material when gas supply pressure is less than $0.4 \mathrm{MPa}$, it is convenient to construct, and it has advantage of good anti-corrosion property and hydraulic conditions and lower cost, so SP are replaced gradually by PEP in practical engineering when the gas supply pressure is not high. For the application case, selecting PEP as the optimal gas pipeline material is reasonable. 
Table 6. Pipeline Materials Function Grades and Function Coefficients

\begin{tabular}{ccccc}
\hline Index (B) & Index (C) & SP & DIP & PEP \\
\hline \multirow{4}{*}{ B1 } & C1 & 9 & 8 & 7 \\
& C2 & 6 & 9 & 9 \\
& C3 & 7 & 9 & 9 \\
& C4 & 8 & 7 & 9 \\
& Grade of B1 & 7.8414 & 8.3823 & 7.9964 \\
\hline B2 & C5 & 8 & 7 & 9 \\
& C6 & 7 & 8 & 9 \\
& C7 & 8 & 9 & 7 \\
& C8 & 8 & 9 & 7 \\
& C9 & 9 & 8 & 6 \\
& C10 & 9 & 8 & 7 \\
& Grade of B2 & 7.7138 & 8.2179 & 7.9612 \\
\hline B3 & C11 & 8 & 9 & 9 \\
& C12 & 7 & 8 & 8 \\
& C13 & 9 & 8 & 9 \\
\hline Total grade and & C14 & 7 & 9 & 8.505 \\
\hline function coefficient & Function coefficient & 0.3214 & 0.3440 & 0.1021 \\
\hline
\end{tabular}

Table 7. Pipeline Materials Annual Costs and Cost Coefficients

\begin{tabular}{cccc}
\hline Item & SP & DIP & PEP \\
\hline Investment cost $\left[\times 10^{4} ¥\right]$ & 177.14 & 241.02 & 162.17 \\
Computing time [year] & 25 & 50 & 50 \\
Operating cost $\left[\times 10^{4} ¥ / \mathrm{a}\right]$ & 6.20 & 4.82 & 3.24 \\
Annual cost $\left[\times 10^{4} ¥ / \mathrm{a}\right]$ & 18.78 & 18.03 & 12.13 \\
Cost coefficient & 0.3837 & 0.3684 & 0.2479 \\
\hline
\end{tabular}

Table 8 Value Indexes of Pipeline Materials

\begin{tabular}{cccc}
\hline Pipeline material & SP & DIP & PEP \\
\hline Value index & 0.8378 & 0.9338 & 1.3497 \\
\hline
\end{tabular}

\section{Conclusions}

Based on the VE theory, a model is established used in selection of gas pipeline materials in gas engineering. The model uses value index as the evaluating parameter to evaluate gas pipeline materials, and the the programs and methods are given. The model is used in an application case, and the result shows the model is feasible for selecting gas pipeline material and the evaluation result is intuitive.

According to the actual gas supply projects, the gas pipeline materials function indexes system can be perfected in the future work, and the function indexes can be adjusted with the experts' suggestion to achieve the aim of improving fuctions and reducing cost.

\section{Acknowledgement}

This research was financially supported by the College Outstanding Young Teachers Domestics Visiting Scholar Programs of Shandong Province. 


\section{References}

[1] ZHU Xiaoyun, Cost Control of Gas Pipeline Project, Gas \& Heat, 33(2013)28-30.

[2] YANG Haixiang, WANG Wenxiang and PENG Zhijun, Selection and Practice of Thin-wall Stainless Steel Tube, Gas \& Heat, 34(2014)20-25.

[3] Yang Haixiang, Fan Shuanshi, Application of Fuzzy Comprehensive Evaluation Method in the Selection of Gas Pipe, Urban Gas, 10(2014)16-22.

[4] MILES L D., Techniques of Value analysis and Engineering, McGraw-Hill, New York, 1972.

[5] Florian G. H. Behncke, Sebastian Maisenbacher, Maik Maurer, Extended Model for Integrated Value Engineering, Procedia Computer Sci., 28 (2014)781-788.

[6] YAN Yong-qiang, Application of Value Engineering in Construction Project Management, Value Eng., 9(2015)1-2.

[7] Haiwen Shu, Lin Duanmu, Chaohui Zhang, Yingxin Zhu, Study on the decision-making of district cooling and heating systems by means of value engineering, Renew. Energy, 35(2010)1929-1939.

[8] YANG Xiu-Mei, SUN Yong, WANG Mei-Ji, TIAN Yue, Network User Trust Degree Evaluation Model by Analytic Hierarchy Process, Computer System \& Application, 25(2016)267-270.

[9]JiayouLiu, Yanxin Zhao, Application of Value Engineering in Selection of Villa Air-Conditioning System, 2009 Int. Conf. Inf. Manage. Innov. Manage. Ind. Eng., Xi’an, (2009)515-518.

[10] Boyun Guo, AIi Ghalambor, Natural Gas Engineering Handbook, Gulf Publishing Company, Houston, 2005.

[11] Yuan Deng, Handbook of Gas Planning and Design, China Architecture \& Building Press, Beijing, 1992. 